Tage wurde ein Vortrag gehalten, der hier im Auszug wiedergegeben wird:

„Aus der Geschichte der Herstellung der Panzerplatten in Deutschland“. Von Geh. Baurat Dr.-Ing, e.h. Dr. phil. e. h. Emil Ehrens berger, Traunstein. Der Vortragende fuhrte aus, daß die Bekleidung von schwimmenden Batterien mit $11 \mathrm{~cm}$ dicken Schmiedeeisenplatten im Jahre 1854 durch Dupuy de Lome als der Ausgangspunkt für die Eisenpanzerung der Kriegsschiffe zu betracbten sei. Das erste eisengepanzerte Schiff war die im Jahre 1859 von Stapel gelassene franzosische Fregatte „Gloire“, der rasch englische Schiffe folgten. Die Dicke der anfănglich 80 bis $120 \mathrm{~mm}$ dicken Panzerplatten wuchs gleichzeitig mit der Durchschlagskraft der Geschuitze, und bald entstanden Panzerstärken von 55 und selbst $60 \mathrm{~cm}$. Aus Gründen der leichteren und besseren Herstellung wurden derartige Panzer auch geteilt, in zwei hintereinanderstehenden Platten angeordnet, was man als „Sandwich"-Panzerung bezeichnete. Das große Gewicht der Platten zwang dazu, erhebliche Teile der Schiffe ungepanzert zu lassen, und man schien an einem gewissen Endpunkte angelangt zu sein, als es im Jahre 1876 der Firma Schneider \& Cie. in Creuzot gelang, Stahl an die Stelle des Schweißeisens zu setzen, wodurch bei gleicher Widerstandsfähigkeit eine Gewichtsersparnis von $30 \%$ erzielt werden konnte. Aber diese Platten waren sehr spröde und zerbrachen leicht beim Auftreffen der Geschosse. Besser verhielten sich in dieser Beziehung die im Jahre 1877 in England aufgekommenen „Compound " Panzerplatten, deren volderes Drittel aus hartem Stahle bestand, der durch Aufgieben auf die weißglühende Eisenplatte mit dieser verschweißt worden war; sie zeigten ungefähr die gleiche Widerstandsfähigkeit gegen Durchschlagen wie die Schneiderschen Platten.

Die ersten deutschen Kriegsschiffe waren aus England bezogen, und man hatte es der Energie des Admirals v. Stosch zu verdanken, daf nicht nur die weiteren Kriegsschiffe in Deutschland gebaut, sondern auch die Panzerplatten im Lande selbst erzeugt werden konnten. Die Dillinger Huttenwerke nahmen im Jahre 18i7 die Herstellung von Schmiedeeisenplatten auf, führten aber bereits im Jabre 1880 die englische Compoundplatte ein.

Als im Jahre 1890 mit dem Bau der Linienschiffe der Brandenburg-Klasse ein erheblich erweitertes Flottenbauprogramm einsetzte wurde die Firma Krupp zur Aufnahme der Plattenherstellung veranlaßt. Sie nahm im gleichen Jahre die Herstellung auf und lieferte zunächst ebenfalls Compoundplatten, während sie gleichzeitig Versuche ulu deren Verbesserung anstellte. Schon im Jahre 1892 konnte eine von Krupp and Dillingen in gemeinsamer Arbeit geschaffene Platte aus weichem Nickelstahl an die Stelle der Compoundplatte geselzt werden, welche die Widerstandsfähigkeit einer um $16 \%$ dickeren Compoundplatte erreichte und von außerordentlicher Zähigkeit war so daß sie auch nach Beschießung mit sehr zahlreichen Schüssen aus großen Kalibern keinerlei Risse zeigte. Im gleichen Jahre fertigte Krupp eine Platte aus mittelhartem Nickelstahl, die durch Härten in $O 1$ und darauffolgendes Anlassen noch weiter verbessert worden war. Im Jahre 1893 konnte Krupp bereits eine durch Zementation mit Leuchtgas auf der Vorderseite aufgekohlte und sodann unter der Wasserbrause gehärtete Platte auf die Weltausstellung nach Chicago senden, welche damals großes Aufsehen erregte. Diese Nickelstahlplatte kam aber nicht zur Lieferung, da Krupp im Jahre 1894 durch Anwendung von Nickelchromstahl und durch eigenartige Härtung der ebenfalls mit Leuchtgas zementierten Platte ein wesentlich besseres Erzeugnis an die Stelle setzen konnte. Diese sogenannte Krupu-Platte zeichnete sich durch auBerordentlich große Widerstandsfähigkeit aus, derart, daß sie einer dreimal so dicken Schmiedeeisenplatte als gleichwertig gegen Durchschlagen zu erachten war. Zudem wur sie von einer unvergleichlichen Zäbigkeit, so daß sie mit zahlreichen Schüssen belegt werden konnte, ohne irgendwelche Risse zu zeigen. Diese Platie nun wurde von allen Marinen der Welt eingeführt und ist bis heute in Gebrauch geblieben.

Der Vortragende schloB an die al]gemeinen Ausführungen über den Entwicklungsgang der Panzerplatten eingehende Schilderungen des Herstellungsverfahrens der „Krupp-PJatte ${ }^{*}$ in metallurgischer und technischer Beziehung an. Der "Ausgangsstoff für die "Krupp-Platte war ein im basischen Siemens-Martin-Ofen hergestellter Nickelchromstahl mit einem Gehalt von $0,35 \%$ Kohlenstoff, $4 \%$ Nickel und $1,75 \%$ Chrom. Der gegossene Block wurde auf die verlangte Platten. dicke ausgewalzt. Sodann kam die Platte zur Zementation, wobei uber die auf rund $1000^{\circ}$ erhitzte Plattenoberfläche 10 bis 15 Tage lang Leuchtgas geleitet wurde, das seinen Kohlenstoffgehalt an den Stahl abgab, so daß eine Anreicherung des Kohlenstoffgehaltes der Platte bis zu $4 \mathrm{~cm}$ Tiefe und bis zu 1,5\% erreicht wurde. Die zementierte Platte wurde dann sofort zum Zwecke der Gefügeverfeinerung in Rubol abgeloscht, worauf eine Erhitzung auf $630^{\circ} \mathrm{mit}$ nachfolgender Abkukhlung in Wasser folgte. Durch dieses Verfahren war der Platte die hocbste ihr zu erteilende Zähigkeit gegeben. Nun setzte die Formgebung unter einer 10000-t-Biegepresse und die mechanische Bearbeitung der Platte ein. Es handelte sich dann noch um die Härtung der Platte, zu welchem Zweck die Vorderseite allein der Wirkung strahlender Wärme so lange ausgesetzt wurde, bis sie die Härtetemperatur von durchschnittlich $870^{\circ}$ erreicht hatte, wăhrend die Plattenrickseite vor Wärmezufuhr geschutzt wurde, um die ihr bereits erteilte Zăhigkeit nicht herabzumindern: Die solcherart "differentiell" erhitzte Platte hărtete man durch plostzliche Abktuhlung unter einer starken Wasserbrause. Die Vorderseite war dann härter als Glas, die auf die Platte verfeuerten Geschosse wurden zerschmettert: -

Nach Beendigung des Vortrages wurde Herrn Ehrensberger als dem Bahnbrecher bei der Herstellung und Auswertung hochwertiger Stăhle die Carl-Lueg-Denkmünze des Vereins ubbergeben.

Dozentenverband. Am 10./12. 1921 vereinigte sich eine großere Zahl von Dozenten für Chemie an Südwestdeutschen Hochschulen in Darmstadt. Bei diesem Anlasse wurde die Bildung eines losen Dozentenverbandes zur Sprache gebracht. Es wurden eine Reihe wissenschaftlicher Vorträge gehalten. Es sprachen: Freúdenberg, Freiburg: "Uber Catechin". - Stollé, Heidelberg: "Uber die Umsetzung von Dihydracidchloriden mit Natriumacid ${ }^{\star}$. - S ch warz, Freiburg: Uber die Beeinflussung der Wasserstoffsuperoxydkatalyse durch Röntgenstrahlen“. - B rigl, Tubingen: "Uber die Reduktion eines Flavonfarbstoffs zum Anthocyan“. - v. Halban, Würzburg: "Über die Verwendung photoelektrischer Zellen zur Messung der Lichtabsorption". - Schoeller, Freiburg: Uber die biochemische BedeutungorganischerQuecksilberverbindungen".- Gold s c h m id t, Würzburg: "Zweiwertiger Stickstoff: Uber Hydracyle“. - v. Br a un, Frankfurt a. M.: "Zur Kenntnis der Alkamine".

\section{Neue Bücher.}

Die Meldepflicht der Berufskrankheiten. Eine Umfrage, bearbeitet von Dr. Francke, Frankfurt a. M. und Dr. Bachfeld, Offenbach, Berlin 1921, Julius Springer, 49 Seiten.

Die vorliegende Schrift stellt das Ergebnis einer Umfrage dar; es ist das Verdienst des Instituts für Gewerbehygiene in Frankfurt a. M., sie einer großen Anzahl Ärzten, Technikern, Verwaltungsbeamten, Industriellen und Arbeitnehmern unterbreitet zu haben. Das Resultat dieser Fragebogen wird von den Autoren in geschickter Weise zusammengefaßt. Jeder Chemiker, Techniker, Beamter und Arzt, der nur im geringsten in Beziehungen zur Industrio steht und soziales Empfinden hat, sollte sich den Inhalt zu eigen machen und mithelfen, die darin wiedergegebenen Anregungen zu vervollkommnen. Als auf etwas ganz Erschopfendes soll und darf das Resultat dieser Umfrage noch keinen Anspruch machen, es enthält ein gutes Fundament, auf dem aufgebaut werden kann.

Wabrend in Deutschland, als dem industriell hochst entwickelten Staate, die ersten Ansätze zur Meldepflicht der Berufskrankheiten aus dem Jahre 1907 stammen und zwar nur für Berufserkrankungen einzelner Industriezweige (Phosphor, Arsen, Milzbrand), die erst nach und nach auch auf andere Berufserkrankungen ausgedehnt wurden, hat das Ausland, namentlich die Sehweiz und England, die Vorschriften zur Meldepflicht und Entschädigung der Betroffenen weit früher (Schweiz 1901, England 1901 und 1906) viel allgemeiner erfabt und durchgefibihrt.

Die Schrift enthät weiterhin den Wortlaut des Fragebogens, der aus neun kurz und prägnant formulierten Fragen bestand, und die Antworten der Angefiagten.

Der Extrakt dieser Fragen läbt sich vielleicht in die zwei Schlagworte zusammenfassen:

1. Erweiterung der Meldepflicht und ihre Handhabung (Frage 1-4),

2. ärztliche Begutachter und deren sachverständige Ausbildung (Frage 5-9).

Wăhrend sich nur ein geringer Teil der Beantworter für die Erweiterung der Meldepflicht, teils zogernd, die Schwierigkeit der Durchfübrung betonend (R. Fischer, Teleky, Ascher Scheideanstalt), teils ablehnend (Böttrich) und, in jeder Form" ablehnend (Herx heimer) ausspricht, tritt die weitaus grobte Anzahl aller anderen Bearbeiter warm für die Erweiterung ein und bezeichnen sie zum Teil als dringend erforderlich.

Interessant sind die Antworten der nichtärztlichen gegentiber den ärztlichen Bearbeitern bei der Beurteilung der Fragen: Wer soll die Meldung erstatten, und an wen ist sie zu richten? DaB Kombinationen mehrerer Stellen, wie verschiedene zu beiden Fragen vorschlagen, der gangbarste Weg ist, damit eine Meldung die andere kontrolliert, ist für die wissenschaftliche Erforschung der Berufserkrankungen sowohl wie für die Grundlage eventueller Entschädigung der Erkrankten wïnschenswert.

Die weitaus bedeutungsvollere Frage ist die der ärztlichen Begutachtung und die unbedingte Notwendigkeit, hierzu theoretisch und praktisch vorgebildete Ärzte zur Diagnosestellung und Beurteilung zu besitzen. "Hierzu sprechen sich Francke und $B$ achfeld ausführlich aus, und es-seien Interessenten direkt auf die Schrift verwiesen. $D_{d} B$ die Ausbildung der Ärzte auf den Universitäten binsichtlich Erkennung und Behandlung der Berufskrankbeiten eine sehr mangelhafte ist, daruber ist die Mehrzahl einig; dementsprechend fordern die meisten befragten Universitätslehrer Fortbildungskurse nach beendetem Studium. Doch das genügt nicht. Dartiber, daß eine praktische Sonderausbildung wlinschenswert erscheint, besteht hei allen Beantwortern kein Zweifel; desto mehr jedoch uber den Modus eines solchen (S. 30 ).

Die Anregungen Hanauers und Lehmanns (S. 31) dürften dieser Frage die richtige Antwort geben. Nur als Assistenten der Fabrikkrankenhäuser und der Fabrikărzte in industriereichen Gegenden kann den jungen Medizinern die Ausbildungsmóglichkeit in Berufskrankheiten geschaffen werden. In den rein äratlichen Fragen spricht sich Bachfeld noch eingehender aus, er kommt zur Aufstellung von 
Krankheitsgruppen, die als meldepflichtige Infektionen oder als meldepflichtige Folgen der Arbeit aufzufassen sind, und stellt am Schlusse seiner Ausführungen Entwürfe der Anzeigen einer Gewerbekrankheit 1. durch den behandelnden Arzt, und 2. durch den Arbeitgeber oder seinen Beauftragten auf.

Ein nicht hoch genug zu schätzendes Verdienst hat sich das Institut für Gewerbebygiene und insbesondere Francke dadurch erworben, eine Reihe praktisch erfahrener Männer und sachverständiger Gelehrter mit der Materie erneut beschäftigt zu haben. Aber dies darf allein das Endziel nicht sein. Es muß jetzt weiter gebaut werden. Zur Weiterförderung der gewonnenen Resultate gebören Forschungs- und Sammelstätten fär die experimentelle und praktische Gewerbehygiene, und wenn sich solche aus finanziellen Gründen, wie Francke im Schlußworte (S. 48/49) ausführt, heute schwer errichten ıassen, so können solche wenigstens an Bestebendes angegliedert werden. Ebenso wie in Dortmund durch Anschluß an das städtische pathologische Institut ein Forsehungsinstitut für Gewerbehygiene und Unfallkrankheiten entstanden ist, so könnte das Frankfurter Institut für Gewerbehygiene im Verein mit dem Senckenbergischen pathologischen Institut und dem Pharmakologischen Institut der Universität zu einem Forschungsinstitut, vielleicht speziell für die Anilinfarbenindustrie, ausgebaut werden. Der gleiche Gedanke wurde im Mai 1920 in einer Sitzung der Vertreter der Berufsgenossenschaft für die chemische Industrie und der Anilinfarbenindustrie vom Referenten dieses in seinfr Eigenschaft als Mitglied der vom Ärzt]ichen Verein Frankfurt a. M. erwählten Kommission zur Erforsrhung der bösartigen Blasengeschwülste angeregt und insbesondere darauf hingewiesen, wie wichtig eine Sammelforschung über alle Anamnesen der in Deutschland alljährlich an bösartigen Blasengeschwülsten Verstorbenen für die gesamte Krebsforschung wäre. Der anwesende Vertreter der Regierung, Herr La y m an $n$, hatte zugesagt, eine derartige Sammelforschung in die Wege zu leiten. Näheres darüber ist bisher nicht veröffentlicht worden.

Wie schon eingangs erwähnt, kann das durch die Umfrage gewonnene Resultat nicht als ganz vollkommen betrachtet werden. Es sei mir daher gestaftet, auf ein Symptom entstehender Berufskrankheit hinzuweisen, das in der vorliegenden Schrift ganz unbeachtet geblieben ist. Herr Dr. Fra ncke, Leiter des Instituts für Gewerbehygiene zu Frankfurt a. M., wird den chemischen und ärztlichen Kollegen für weitere diesbezügliche Anregungen sicherlich auch dankbar sein.

Wohl wird von Bachfeld als Folgen gewerblicher Arbeit als meldepflichtig in seinen Entwürfen aufgestellt (S. 41/43) Blasengeschwälste bei Arbeitern in chemischen Fabriken und Färbereien, auch wenn diese Arbeiten schon zehn oder mehr Jahre zurïckliegen“. Diese Fassung gen ügt nicht!

Wie Leuenbergerl) und Referent dieses ${ }^{2}$ ) nacbgewiesen haben, beginnen alle bösartigen Blasengescbwülste, die oft nach 10-15 Jahrea als solche erst zum Ausbruch kommen, mit Blutharnen.

Für alle Arbeiter, die in für Blasengeschwülste gefährdeten $\mathrm{Be}-$ trieben arbeiten, ist die Forderung aufzustellen, den Urin in achttägigen Intervallen $\mathrm{zu}$ untersuchen (Zeitschr. f. angew. Chemie 1919 S. 331, sub 3), und sie sind beim Vorhandensein von Blutharnen (wenn andere Erkrankungen des Urogenitalsystems ausgescblossen sind) als berufskrank zu betrachten, damit die betroffenen Arbeiter oder deren Familien bei einem nach $10-15$ Jahren auftretenden Blasengeschwulst einer Entschädigung nicht verlustig gehen. Diese Feststellurg ist wichtig, weil über $20 \%$ der Arbeiter in Anilinfarbenfabriken nicht seßhaft sind, weiterwandern und hierbei den Keim zu Blasengeschwälsten in sich tragen können. Es ist nachgewiesen, daß eine Anzahl Arbeiter in der ländlichen Heimat 10-15 Jahre nach ihrer Arbeitstätigkeit in chemischen Fabriken an Blasencarcinom erkrankten und, von Landärzten nicht erkannt, eines elenden Siechtums dahinstarben.

Das gleiche gilt von okkulten Blutungen des Magendarmtraktus, die, wie Lew in mitgeteilt bat, hei Intoxikationen mit Kohlenwasserstıffen (insbesondere Benzol) auftreten können. Alle Erkrankungsfälle mit Auftreten von Blut in Harn oder Faeres, unter vorstehenden Voraussetzungen, müssen daher ehenfalls als eine Folge gewerblicher Arbeit angesehen und zur Anmeldung gebracht werden.

Dr. Max Nassauer, Frankfurt a. M. [BB. 24.]

Leitfaden der Physik und Chemie. Für die oberen Klassen von Bürgerschulen, Mittelschulen usw. Von A. Sattler. 45. u. 46. verbesserte ii. vermehrte Auflage. VIII u. 283 S. 321 Abbildungen. Friedr. Vieweg u. Sohn, Braunschweig, 1921. geb. M $16+20 \%$ Verl.-Zschl.

Der - gegenüber dem physikalischen stiefmütterlich behandelte chemische Teil dieses Buches gibt die ja leider in so vielen Schulbüchern übliche nüchterne Aneinanderreihung wissenschaftlich-chemischer Tatsachen. Über didaktische Schwierigkeiten setzt sich der Verfasser leicht hinweg (vol. die Ausführungen über Molekül, Atom, Element auf den Seiten 173 und 174). Der Schüler bekommt weder ein Bild von der Bedeutung der Chemie für Natur und Leben, noch wahres Verständnis für die Grundlagen unserer chemischen Anschauungen. Alfred Stock. [BB. 224.]

1) Leuenberger: Die unter dem Einfluß der synthetischen Farbenindustre beobachtete Geschwulstbildung. Beitr. z. klin. Chirurgie 80, 1912.

2) Nassauer: Über bösartige Blasengeschwülste bei Arbeitern der organisch-chemischen Großindustrie: Frankfurter Zeitschr. f. Pathologie, Bd. 22, Heft 3, und Zeitschr. für angew. Chemie, 32, 333 [1919].
Organic Compounds of Mercury. Von C. Frank, Whitmore, Ph. D. Verlag The Chemical Catalog Compuny, Ine. One Madison Avenue, New York, U. S. A.

Das vorliegende Buch, welches einen Band der von der Amerikanisch - Chemischen Gesellschaft herausgegebenen Monographiensammling bildet, bebandelt die Chemie der organischen Quecksilberverbindungen mit einer Ausführlichkeit, wie sie, mit Rücksicht auf die Tendenzen dieser Sammlung, wissenschaftliche oder technische Probleme nach ihrem jeweiligen Entwicklungsstand in allen Einzelheiten darzustellen, von vornherein erwartet werden durfte. Nach einer kurzen historischen Übersicht werden zunächst die hauptsächlichsten Herstellungsmethoden der organischenQuecksilberverbindungen beschrieben und daran anschließend ihre generellen Eigenschaften und die chemischen Umsetzungen besprochen, zu welchen diese Verbindungen befähigt sind. Weiter kommen alsdann in getrennten $\mathrm{Ab}$ schnitten die verschiedenartigen, durch Quecksilber substituierten $\checkmark$ erbindungstypen zur Bebandlung, indem jeweils in der vornehmlich in deutschen Publikationen üblichen Weise Herstellung und Eigenschaften, auch der Einzelverbindungen, besehrieben werden. Die Art der Darstellung ist deshalb klar und anschaulich. Die Literatur, und zwar in erster Linie die deutsche, ist bis 1920 berücksichtigt.

In einem Anhang wird sodann ein kurzer Überblick über die Analyse der organischen Quecksilberverbindungen geboten und weiterhin eine Zusammenstellung über die in den Arzneischatz übernommenen Quecksilberpräparate gegeben, ohne daß der Verfasser jedoch, von gelegentlichen und in dem ganzen Buch verstreuten Hinweisen $a b$ gesehen, auf ihre therapeutische Bedeutung des näheren eingeht. Ein Literaturnachweis und eine Zusammenstellung der in den verschiedenen Kulturstaaten auf dem Gebiete der organischen Quecksilberverbindungen entnommenen Patente bilden neben dem üblichen Sach- und Namenregister den Schluß des Buches.

Das Werk, welches auf dem in Frage kommenden Gebiete eine füblbare Lücke ausfüllt, durf vornebmlich all denen zur Anschaffung empfohlen werden, die sich mit der chemischen Synthese organischer Quecksilberverbindungen beschäftigen, wiewohl es naturgemäß gerade hier interessiert, neben dem rein chemischen Tatsachenmaterial auch die Prinzipien kennen zu lernen, auf denen speziell die therapeutische Wirkung und die unter Umständen starke Desinfektionskraft dieser Verbindungen beruhen.

Hoffentlich bleibt es deutschen Forschern vorbehalten, das Buch in Kürze auch in dieser Richtung zu ergänzen.

$$
\text { Dr. Schrauth. [BB. 160.] }
$$

\section{Personal- und Hochschulnachrichten.}

Prof. Dr. Paul Pfeiffer, Karlsruhe, hat den Ruf auf den Lehrstuhl der Chemie an der Universität Bonn, als Nachfolger des Prof. R. Anschütz angenommen (vgl. d. Ztschr. 34, 376 [1921]).

Es wurden berufen: Dr. W. Dilthey, Privatdozent fur Chemie an der Universität Erlangen, an die Tecbnische Hochschule Karlsruhe; Dr. E. Müller, Prof. an der Universität Köln, als a. o. Prof. für Chemie an die Universität Heidelberg als Nachfolger des verstorbenen Prof. E. Knoevenagel.

Prof. Dr. L. Michaelis, Berlin, hat seine Stellung als Bakterio'loge am Städtischen Krankenhaus am Urban aufgegeben und die Leitung des von den "Vereinigten Fabriken für Laboratoriumsbedarf" eingerichteten Biochemischen Instituts übernommen.

Gestorben sind: Dr. phil. L. Kuckro, am 26.12. zu Wiesbaden, im 74. Lebensjahre. - Dr. K. Kuhn, Fabrikbesitzer in Nürnberg, vor kurzem.

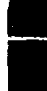 \\ Nach einem arbeitsreichen Leben verschied am 28. Dezember 1921 der Seniorchef meiner Firma, \\ der Kgl. Sächs. Kommerzienrat Herr Heinrich Theodor Koerner Ritter pp. \\ nach nur eintägigem Kranksein.}

Was der Verstorbene in 23 jähriger Inhaberschaft seiner Firma gewesen ist, was er geleistet und geschaffen hat, wird unvergessen bleiben.

Ehre seinem Andenken!

Chemnitz, den 31. Dezember 1921.

Tintenfabrik Eduard Beyer Chemnitz 